\title{
Development of a Clinically Relevant Animal Model for the Talar Osteone- crosis in Sheep
}

\author{
Chao-Fan Yuan', , Jun-Lin Wang 2,, Yong-Quan Zhang', Xiao-Kang Li', Yi Li', Su-Hua Wu', Zhi-Yong \\ Zhang $^{3 凶}$, Zheng Guo ${ }^{1 凶}$
}

1. Department of Orthopaedics, Xijing Hospital, Fourth Military Medical University, Xi'an, China;

2. School of Stomatology, Fourth Military Medical University, Xi'an, China;

3. Department of Plastic and Reconstructive Surgery, Shanghai 9th People's Hospital, Shanghai Key Laboratory of Tissue Engineering, School of Medicine, Shanghai Jiao Tong University, Shanghai, China.

* The authors have contributed equally to this paper as co-first authors for this article.

\begin{abstract}
$\triangle$ Corresponding author: Guo Zheng, MD, PhD. Mailing Address: Department of Orthopaedics, Xijing Hospital, Fourth Military Medical University, No. 127, Changle West Road, Xi'an, Shaanxi Province, 710032, P.R.China. Tel: 86-29-84773411,Fax: 86-29-84773411 E-mail: guozheng@fmmu.edu.cn or Zhang Zhi-Yong, PhD. Mailing Address: Department of Plastic and Reconstructive Surgery, Shanghai 9th People's Hospital, Zhizaoju Road, No. 639, Huangpu District, Shanghai, 200011, P.R. China. Tel: 86-21-23271699, Fax: 86-21-63136856, E-mail: mr.zhiyong@gmail.com.
\end{abstract}

(C) Ivyspring International Publisher. This is an open-access article distributed under the terms of the Creative Commons License (http://creativecommons.org/ licenses/by-nc-nd/3.0/). Reproduction is permitted for personal, noncommercial use, provided that the article is in whole, unmodified, and properly cited.

Received: 2012.07.16; Accepted: 2012.10.15; Published: 2012.10.27

\begin{abstract}
There are a lot of reports and reviews about osteonecrosis of the talus (ONT), yet reports about the animal model of ONT to evaluate proper therapeutic approaches are rarely heard. In our study, a novel animal model was established. Pure ethanol was injected into the cancellous bone of sheep's talus. Macroscopic observation, X-ray, CT and histology were performed at two, four, 12 and 24 weeks postoperatively. It was revealed that the trabeculae of talar head began to change their structure after two weeks postoperatively compared to the normal talus. The ONT was obvious at the end of the fourth week, and their outstanding feature was the damage of trabeculae bone and formation of cavities. CT scans and pathological changes of the subjects all showed characteristics of the early stage of osteonecrosis, also the sections of the specimens confirmed necrosis of tali. By 12 weeks, the phenomenon of necrosis still existed but fibrous tissue proliferated prominently and bone reconstruction appeared in certain area. Most specimens (3/4) got late stage necrosis which presented as synarthrosis in X-ray and mass proliferation of fibrous tissue in histology at the end of 24 weeks. The novel animal model of ONT was successful, and it is inclined to deteriorate without any intervention. The study provides us a new way to evaluate various treatments on ONT in laboratory, which may eventually pave way to clinical applications.
\end{abstract}

Key words: Animal model; Osteonecrosis of the talus; Ethanol; Injection.

\section{INTRODUCTION}

Osteonecrosis of the talus (ONT) is a degenerative skeletal disease accompanied with continuous pain and dysfunction of the ankle, which can seriously compromise the routine work and physical activities of patients. ONT could be easily caused by some traumatic ankle injuries such as fractures and dislocations or some untraumatic diseases including systemic lupus erythematosus, scleroderma, insulin-dependent diabetes mellitus, multiple sclerosis, alcohol abuse, heavy smoking and so on [1-5]. Most 
ONTs have generally experienced a phase of ischemic injury after the disturbance of blood circulation, although the detailed pathophysiological process remained unknown [6-8]. Many clinical reports have identified the characteristics and treatments of ONT by imitating the finding and knowledge from osteonecrosis of femoral head, due to its extensive clinical and experimental research, especially in terms of the radiographic diagnosis of disease stages and the therapeutic principles. Currently, most of the ONT research were concentrated to the clinical studies, which have the limitation to treat ONT even in the early stage, because the onset of ONT is gradual and subtle, particularly those untraumatic cases almost have been going on six months before any symptoms appeared [2,4]. Once the osteonecrosis features like cystic changes and crescent sign were clear in X-ray photographs, they often suggested that the actual necrosis in talus has become quite severe. Therefore, it is urgent to conduct more fundamental investigation to understand the mechanism and characteristics of ONT disease and explore the potential treatment strategy for ONT, especially in the early stage.

To develop an animal model of a certain disease is crucial and a highly helpful approach in current biomedical research because the animal model may imitates the nature pathological process of the disease in human being and offers a platform to understand the mechanism of disease in order to explore better diagnostic and treatment strategies. Although a large amount of clinical papers about ONT have been published focusing on diagnosis, conservative and surgical treatments, the experimental research of ONT are generally rare, especially there is no animal model of ONT established so far.

Therefore, in current study, we aim to develop a proper ONT disease model in sheep to provide a platform for investigating the characteristics and treatments of ONT, especially in the early stage.

\section{METHODS AND MATERIALS}

\section{Animals}

All animal experiments were performed in strict accordance with protocols approved by the Institutional Animal Care Committee of the Fourth Military Medical University. Sixteen adult female Small Tailed Han Sheep (median age, 18 months; range, 16 to 24 . median weight, $42 \mathrm{~kg}$; range, 36 to 54 ) were used in the study. Sheep were housed in three approved shacks with the average acreage of $18 \mathrm{~m}^{2}$ in the Orthopaedics of Xijing hospital Animal Resources Center and were maintained under routine husbandry.

\section{Experimental design}

The sheep were weighed and then numbered randomly. All sheep were evenly divided into four experimental groups for examination by the end of two, four, 12 and 24 weeks. The right hind legs of sheep were chosen to induce necrosis model and their intact left tali were set as control group (Figure 1). The sheep were acclimated to their environmental conditions for at least seven days, and then surgical operations were carried out at the medial head of tali. X-ray photographs were taken at every time point postoperatively. After harvesting, five talar specimens were selected randomly from different time points for Computed Tomography (CT) scan, and the three-dimensional images of CT data were reconstructed (threshold: 226-3071 HU) using MIMICS software (Materialise, Belgium). Section observation and examination of histology of the tali were performed accordingly. 16 right tali of all experimental group and four untreated left tali as the control group were harvested at the end of two, four, 12 and 24 weeks postoperatively for detailed analysis (Figure 1).

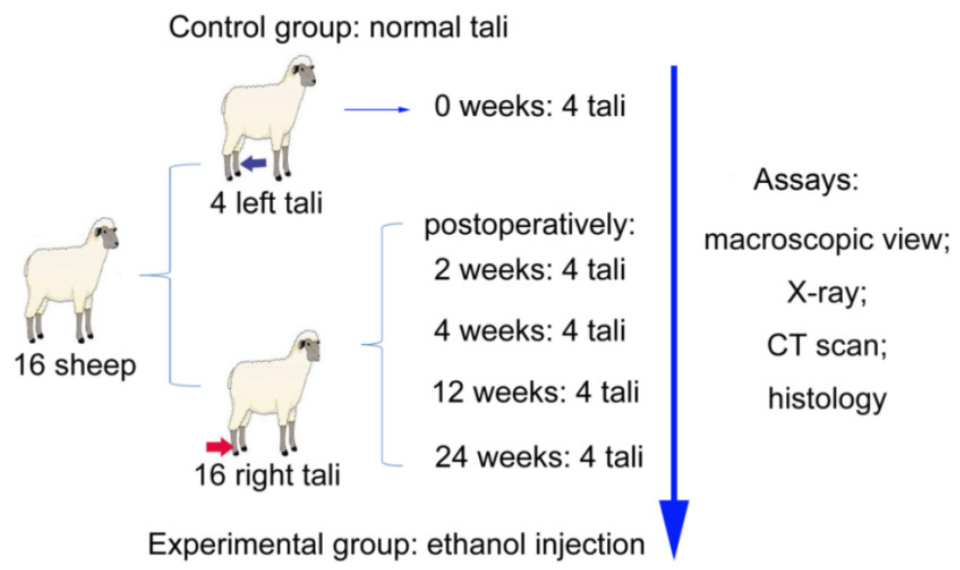

Figure I. Schematic illustration of experimental design. 


\section{Anesthesia and surgical procedures}

The sheep were fasted $24 \mathrm{hrs}$ before surgery. They were anesthetized with Xylazine Hydrochloride Injection $0.2 \mathrm{mg} / \mathrm{Kg}$ (Huamu Animal Health Product Co LTD, Changchun, Jilin, China) intramuscularly through erector spinae, and then atropine sulphates $0.05 \mathrm{mg} / \mathrm{Kg}$ (Xiyue Pharmaceutical Co LTD, Xi'an, Shaanxi, China) were injected subcutaneously to prevent the excessive secretion. After anesthesia the sheep were banded on a custom timber frame, and their tongues were pulled out gently with a tissue forceps in case of asphyxia. Intravenous route was established and Cefazolin Sodium (Harbin Pharmaceutical Group, Harbin, Heilongjiang, China) was transfused into animals at the dosage of $30 \mathrm{mg} / \mathrm{Kg}$ before surgery.

The operation carried out in an independent room under sterile conditions. Compound Amionprine and antipyrine Injection $10 \mathrm{ml} / \mathrm{sheep}$ (Yabao Pharmaceutical Group, Ruicheng, Shanxi, China) was injected intravenously to ease pain and enhance the effect of anesthesia during surgery. The sheep were placed in a lateral position. The region of the medial ankle was sheared and the skin was cleaned with soap. After the skin was disinfected with iodine solution, sterile drapes were placed in layers. Then, the region of the medial ankle was approached by a direct incision about $4 \mathrm{~cm}$. Soft tissue and fascia were separated to expose the joint capsule, and capsule was carefully dissect without damaging the medial tendons in order to expose the medial head of talus. A hole with $1.05 \mathrm{~mm}$ in diameter and $15 \mathrm{~mm}$ in depth was drilled at the central of medial talar head from the medial side to avoid damaging the cartilage surface. Next, $4 \mathrm{ml}$ pure ethanol (Fuyu Corporation, Tianjin, China) was slowly $(0.8 \mathrm{ml} / \mathrm{min})$ injected into talar head through the hole. The hole was sealed with bone wax after injection to prevent the reflux of ethanol. Then the surgical region was irrigated with hydrogen peroxide and saline solution successively and the wound was closed in layers. The antibiotics and analgesics were used for another three days postoperatively.

\section{Histological examinations}

The talar samples were fixed in $10 \%$ formalin for two weeks and decalcified for another two weeks, then the talar heads were cut off. To evaluate the extent of necrosis, we developed a histological appraisal system: the talar heads of all the specimens were divided into four parts through the drill track, so eight sections formed and the intermittent sections marked dark were chosen to get the full view of the necrosis
(Figure 2). All of the divided samples were embedded into paraffin. The selected sections were cut to $5 \mu \mathrm{m}$ thick and stained with hematoxylin and eosin. Then inspired by the method of Levin et al, the bone trabeculae with nucleus less than $1 / 3$ size of the lacunae or empty lacunae were considered to be the necrotic trabeculae and the percentage of the living trabeculae was evaluated in each section [9]. The presence of newborn bone and fibrous tissue were graded as follow (none:-, mild: + , moderate: ++ , much: +++ ).

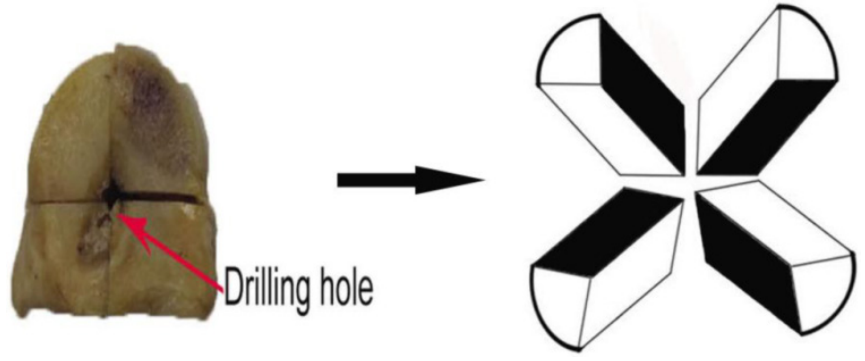

Figure 2. Region of interest for histological study.

\section{Statistical analysis}

The statistical analyses of different timing (percentages of living trabeculae) were performed using SPSS16.0 with univariate analysis of variance. The difference was considered significant at a value of $\mathrm{p}<0.05$.

\section{RESULTS}

The sheep showed good physical condition after surgery with little swell and lameness in the ankle, and they restored normal walking with obvious swell two weeks postoperatively, however, two sheep showed apparent lameness after 20 weeks (Table 1). The extent of swell and lameness of the operated ankle was graded as follow (none:-, mild: + , moderate: ++ , much: +++$)$. Superficial infection was observed in only one sheep, and the sheep recovered in two weeks after careful debridement. There were no other complications occurring throughout the whole study.

\section{Macroscopic examination}

The contour of the cartilage remained intact in all specimens, and the early stage specimens (two weeks) showed several dark parts at both the head and body of tali compared to the normal talus, moreover, the surrounding bone tissue around the drilling entrance experienced partial necrosis, as evidenced by displaying the dark areas of $5 \mathrm{~mm} \times 4 \mathrm{~mm}$ in the cross-section view of specimens with initial disruption of trabecular structure compared to the normal talus (Figure 3A and B). The necrosis of bone tissue progressed with time, with a necrotic cavity about $4 \mathrm{~mm} \times 4 \mathrm{~mm} \times 3 \mathrm{~mm}$ observed inside three out of four 
the talar head samples at week four postoperatively (Figure 3C). However, the size of necrotic cavities were reduce to the size of about $2 \mathrm{~mm} \times 3 \mathrm{~mm} \times 3 \mathrm{~mm}$ with partial regeneration of compact tissue at week 12 postoperatively (Figure 3D). By week 24, many cavities had formed and they interspersed among the sections (Figure 3E).

\section{Radiographic study and CT examination}

At week two, little structure change could be detected compared to normal tali by $\mathrm{X}$-ray imaging (red arrow head, Figure 4A, B and C), nevertheless at the end of the fourth week the change of spongy bone around the drill track became obvious in all specimens, showing lower radiographic density than the normal spongy bone of tali with broken trabecular bone structure (red arrow head, Figure 4D). The X-ray at the $12^{\text {th }}$ week showed little changes compared to the fourth week, and all of the specimens had no remarkable injury on cartilage (red arrow head, Figure $4 \mathrm{E})$. By week 24, the talar head showed deformity of the structure and fused with the adjacent bones (red triangle, Figure $4 \mathrm{~F}$ ). Quantitative analysis of the re- construction by MIMICS showed necrosis led to the formation of necrotic cavities $\left(38.88 \mathrm{~mm}^{3}\right)$ inside the tali two weeks after the ethanol injection (Figure 5B) and with the progress of necrosis, the size of necrotic cavity increased to $105.26 \mathrm{~mm} 3,136.34 \mathrm{~mm} 3$ and 746.05 mm3 with time (Figure 5C, D and E), corroborating the findings of macroscopic images.

\section{Histological analysis}

Histological analysis was performed to further investigate the pathological change of bone tissue at the necrotic site. The normal talus showed regular cartilage and bone trabecular structure, as well as osteocytes in the bone lacunae (green arrow head, Figure 6A). Two weeks postoperatively, many empty lacunae (black arrow head, Figure 6B) were spotted co-existing with osteocytes (green arrow head, Figure $6 \mathrm{~B}$ ) in the trabeculae of cancellous bone, and this is only found in the center of the samples where the necrosis occurs. The osteocytes in the trabeculae of cortical bone and chondrocytes were presented as normal.

Table I. The physical conditions and histological presentation of the sheep: the percentage of living trabeculae of any two groups showed significant differences between the two neighboring time points $(p<0.05)$ except for week 4 vs. week I2 $(p=0.05 I, n=4)$. (Level rank grade: none:-, mild:+, moderate: ++ , much:+++).

\begin{tabular}{|c|c|c|c|c|c|c|}
\hline Time & $\begin{array}{l}\text { Percentages of living trabeculae } \\
(\mathrm{x} \pm \mathrm{s}) \%\end{array}$ & $\begin{array}{l}\text { Difference between two } \\
\text { neighboring time points }(p)\end{array}$ & $\begin{array}{l}\text { Grade of } \\
\text { fibrosis }\end{array}$ & New bone & Swell of the ankle & Lameness \\
\hline Week 0 & $69.67 \pm 1.33$ & & - & - & - & - \\
\hline Week 2 & $52.19 \pm 1.44$ & $<0.001$ & - & - & +++ & + \\
\hline Week 4 & $33.13 \pm 1.57$ & $<0.001$ & + & + & + & + \\
\hline Week 12 & $27.50 \pm 1.88$ & 0.051 & ++ & ++ & - & - \\
\hline Week 24 & $13.75 \pm 1.16$ & $<0.001$ & +++ & - & - & ++ \\
\hline
\end{tabular}

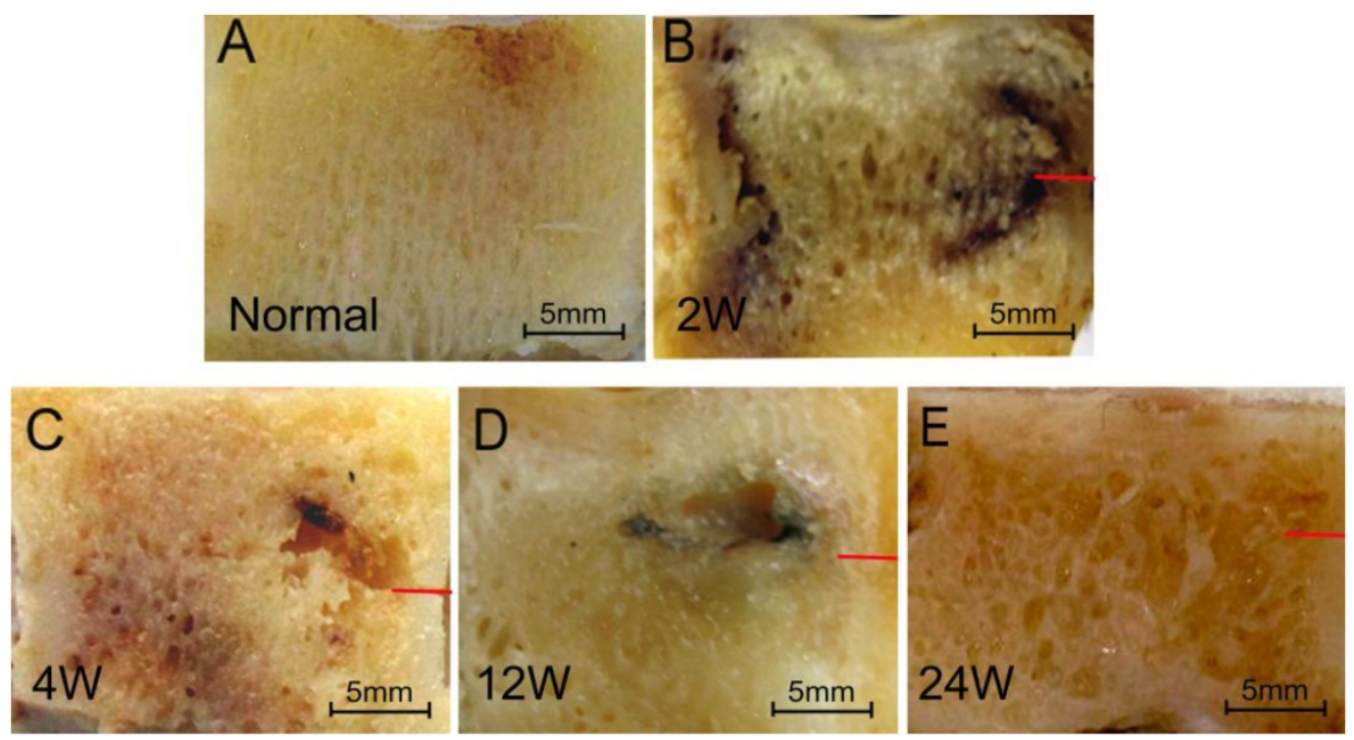

Figure 3. Sections of the talar heads. A: normal talus, displayed an ordered trabeculae structure; B: two weeks postoperatively, trabeculae turned dark in some area with enlarged interval; C: four weeks postoperatively, trabeculae resorbed partially with cavity formed; D: I2 weeks postoperatively, cavity still existed with partial regeneration of trabeculae; E: 24 weeks postoperatively, cavities widely exist in the talus (red bar: the entrance of drilling hole). 

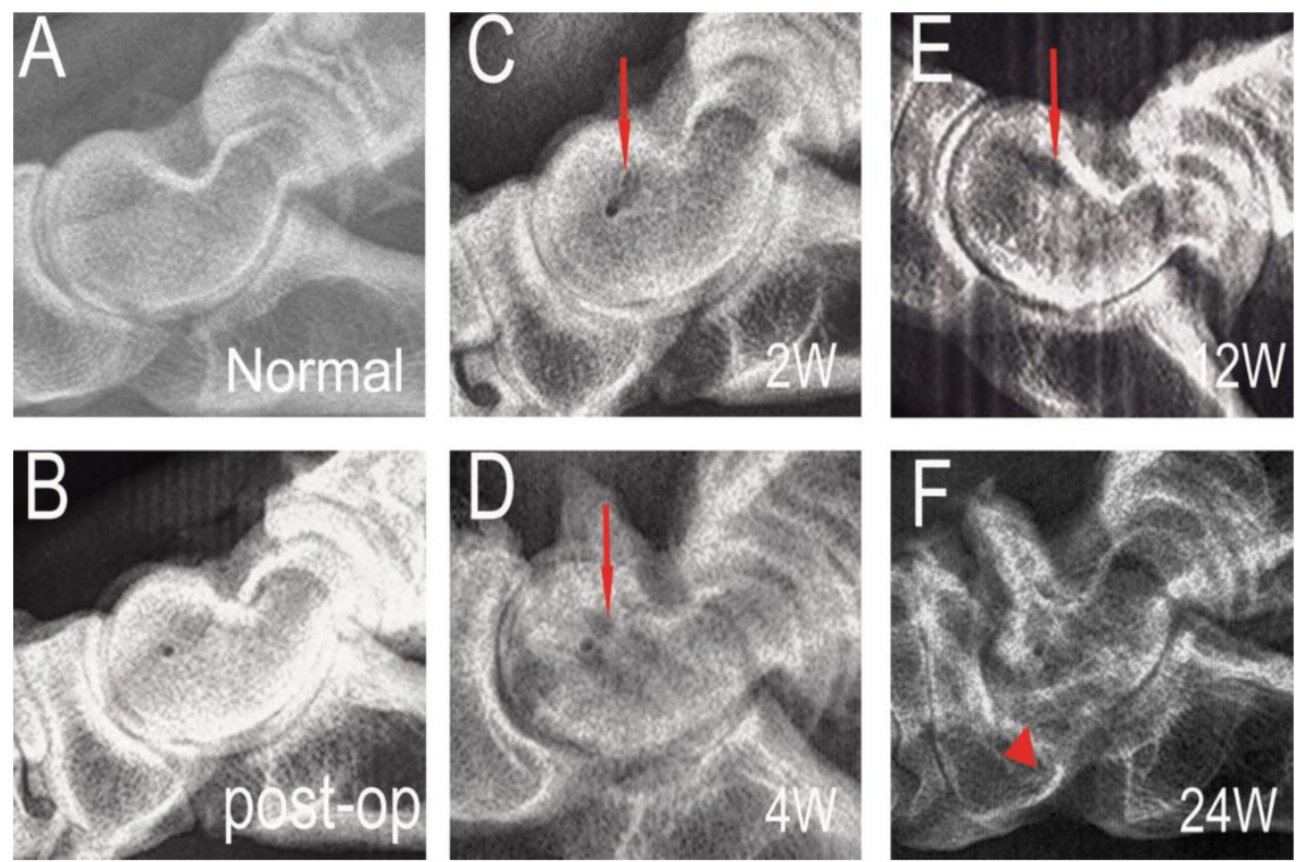

Figure 4. Lateral radiographic images of the talar heads at different time points. A: normal talus; B: immediately after surgery; C: two weeks postoperatively, little change observed around the drilling tunnel (red arrow head); D: four weeks postoperatively, talar head demonstrated a decreased radiographic density (red arrow head); E: 12 weeks postoperatively, talar head displayed a non-homogeneous radiographic density of trabeculae structure (red arrow head); F: 24 weeks postoperatively, the talar head showed deformity of the structure and fused with the adjacent bones (red triangle).

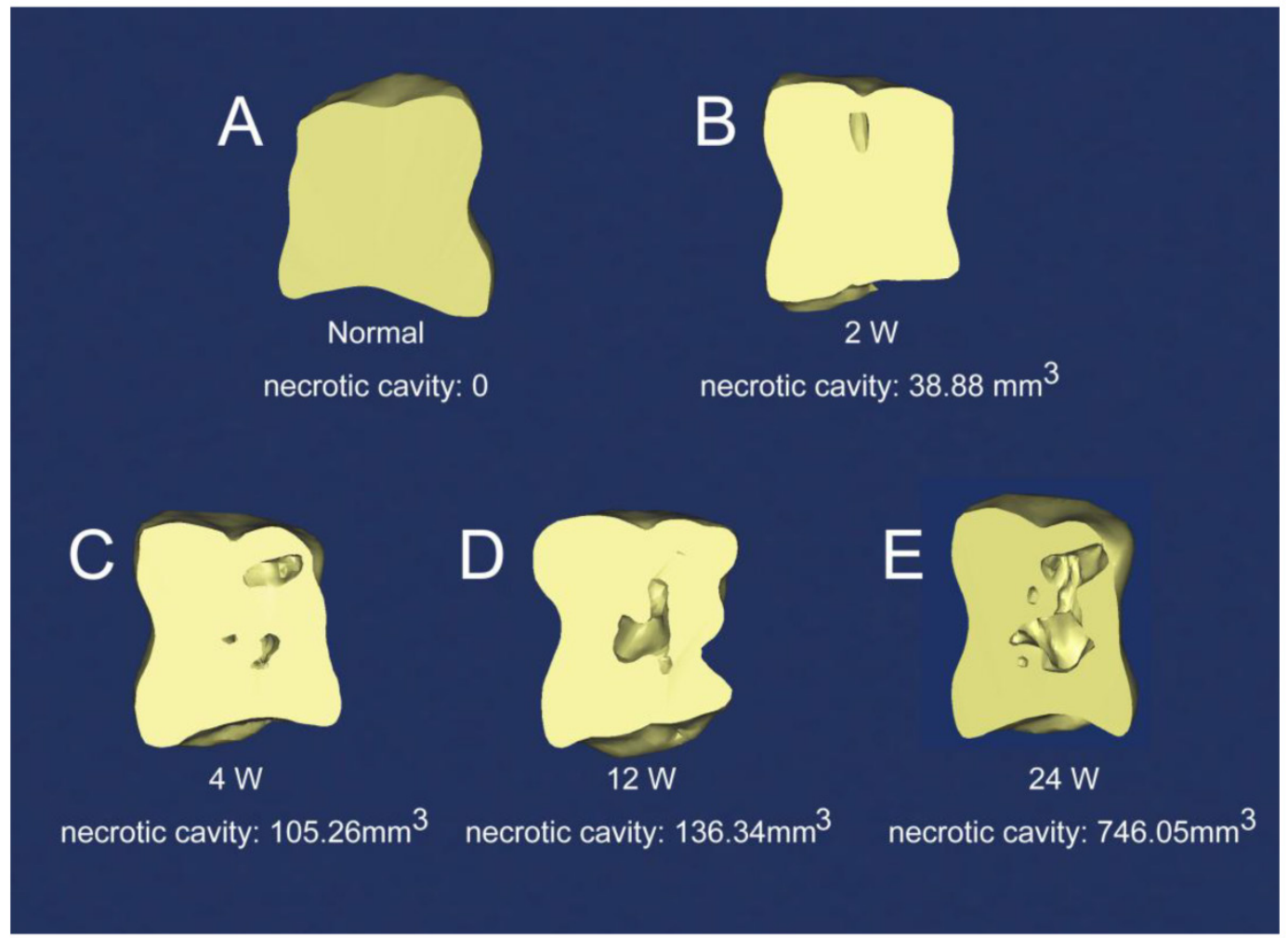

Figure 5. Three-dimensional reconstruction of talar heads by CT data. A: normal talus; B, C, D, and E: necrotic talus at week $2,4,12$ and 24 . 

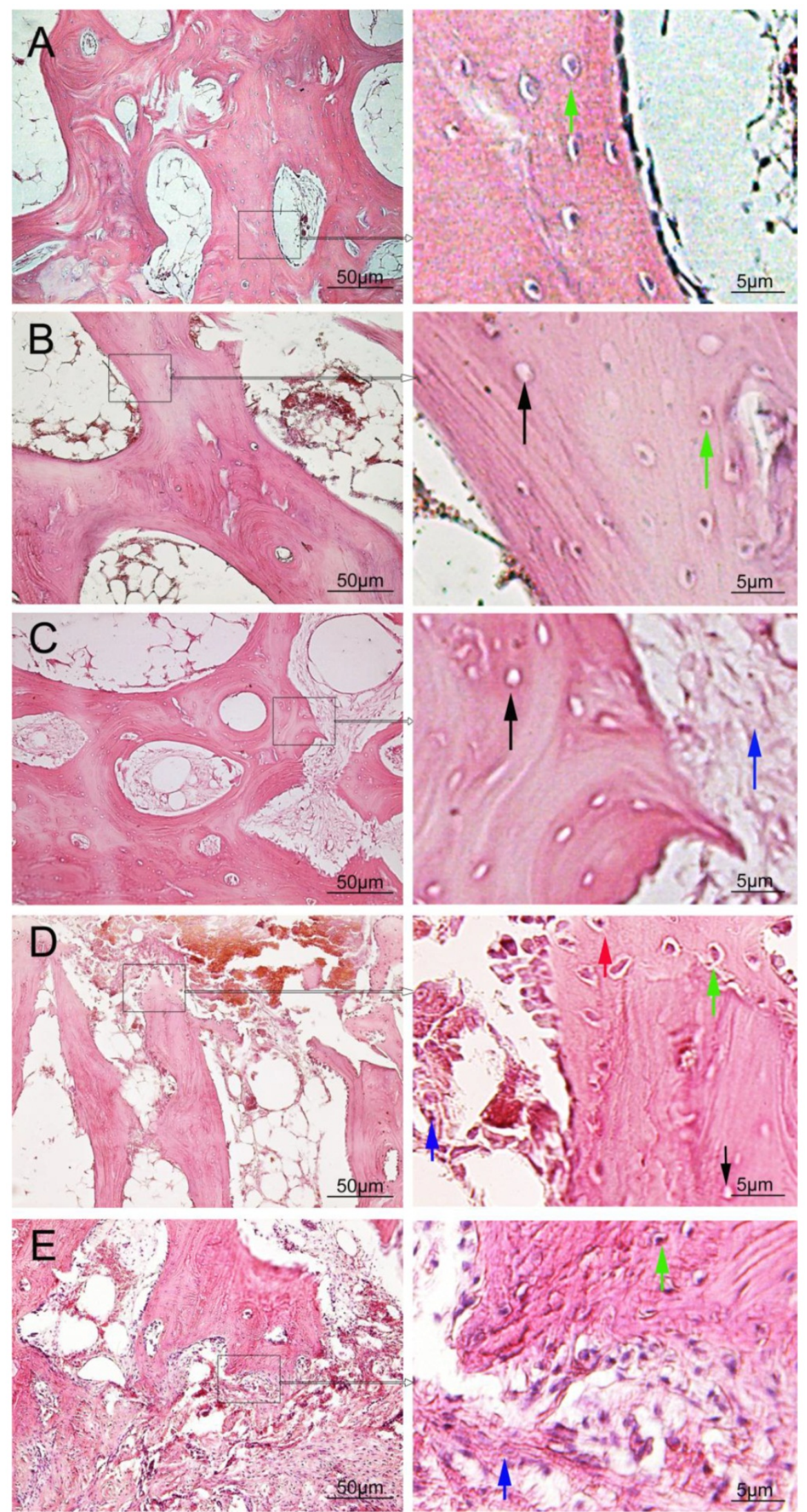

Figure 6. Histological microscopic pictures. A: normal talus; B: necrotic model at two weeks; C: necrotic model at four weeks; D: necrotic model at I2 weeks; E: necrotic model at 24 weeks (hematoxylin and erosin stain $\times 100$, green arrow heads: living osteocytes; black arrow heads: empty lacunae, blue arrow heads: fibrous tissue, red arrow heads: osteoblast and new bone). 
At the end of the fourth week, there were increasing numbers of empty lacunae (black arrow head, Figure 6C) in most bone trabeculae with reduced number of osteocytes, disordered trabecular structure and enlarged gaps. Very limited new bones tissue was regenerated in the edge of several trabeculas with a small amount of fibrous tissues infiltrated into the marrow cavity (blue arrow head, Figure 6C).

At the week 12, large amount of empty lacunae (black arrow head, Figure 6D) still existed in the trabeculae of cancellous bone, but more newly deposited bones together with osteoblasts were found on the edge of trabecular (red and green arrow heads, Figure 6D). Moreover, numerous fibrous tissues were found both around the trabeculae and in the marrow cavity (blue arrow head, Figure 6D).

By week 24, necrotic trabeculas with empty lacunae were rare but the quantity of trabeculas decreased, and there were a great deal of fibrous tissues infiltrated into the marrow cavity (blue arrow head, Figure 6E).

\section{Quantitative analysis}

By using the histological appraisal system, we could quantitatively analyze the necrotic progress of talar bone by calculating the percentages of living trabeculae (Table 1). Osteonecrosis developed rapidly after ethanol injection, with obvious necrotic structure found since week four onward, and less than $1 / 3$ area of the section consisted of living trabeculae structure (Table 1). The percentage of living trabeculae structure decreased quickly with significant difference between two neighboring time points $(\mathrm{p}<0.05)$, except for week 4 vs. week $12(p=0.051)$. In addition, it was found that new bone formation mainly occurred at week 12, and the infiltration of fibrous tissue started at week four and exuberant at week 24 .

\section{DISCUSSION}

To establish a clinically relevant animal model is a key step to study the pathology and investigate the new diagnostic and treatment paradigm of a certain disease. Generally a favorable animal model should fulfill the following requirements: (1) the model resembles the nature of a given disease and could be easily repeated, (2) the selection of the experimental animal is standardized, and (3) the cost of the animals is economical [10]. We choose Small Tailed Han Sheep as the experimental animal because they are purebred sheep and their talar head is very similar to the human talar dome in terms of anatomical structure. Moreover, the biomechanical loading pattern of talar head in sheep is very comparable to human talar dome, as they are both the main load-bearing area of talus [11]. Therefore, we selected the talar head of sheep to develop an osteonecrosis as a clinically relevant large animal model, to study the ONT in human being, which mostly occurred in the talar dome of human.

Osteonecrosis occurs in many parts of the human body, such as the femoral head, the talus, the humerus head, the scaphoid and so on, out of which ONFH (Osteonecrosis of the Femoral Head) is the most intensively disease model. A number of methods have been developed to induce the osteonecrosis in animal model especially for the femoral head. The popular methods include ligation of major vessel in the femoral head, cryogenic treatment with liquid nitrogen, intraosseous destruction with microwave heating, intraosseous injection of pure ethanol and injection of cortical hormone [12-16]. These technologies may shed a light on development of other osteonecrosis models, such as the talus. An ideal technology should be able to induce effective osteonecrosis but not adversely affect the regeneration potential of native bone tissue, because the purpose of research is not only to create a suitable disease model to mimic the clinical settings but also to look for potential treatment methods to cure the disease. Major vessel ligation is a very popular and favorable approach for the development of femoral head osteonecrosis, by inducing successful necrosis without jeopardizing the potential of new bone reconstruction, because the blood supply from the marrow is still enough [17]. However, as far as the talus is concerned, this method may not be so suitable, because the blood supply to talus is limited to three tiny arteries and no supply from marrow. Cryogenic treatment with liquid nitrogen is another popular techniques employed by many researchers to create the osteonecrosis of femoral head $[16,18,19]$. But it usually involve the use of sophisticated equipment and complicated process, moreover, liquid nitrogen might easily destroy the cartilage surface, leading to subchondral collapse. Therefore, the repair based on this animal model is very difficult [18]. Recently, Li YL [13] and his co-workers used microwave heating to create ONFH, although the heating directly destroyed the cancellous bone and formed cystic changes in a short time, this method was more likely to create an animal model of infrabony defect than osteonecrosis, and it could not imitate the pathological process of the early stage of osteonecrosis. Osteonecrosis introduced by cortical hormone was effective for small animals and it seemed not good for big animals, because the large hormone dosage was required [15-20]. The intraosseous injection of pure ethanol could be used to induce necrosis as well. This approach was first de- 
veloped by J. Manggold et al. [14]. The injected ethanol can damage the osteocytes and blood capillary through dehydration, which can lead to the breakage of trabeculae, obstruction of the microcirculation, and eventually the formation of osteonecrosis. After comparison of different approaches, we choose intraosseous injection of pure ethanol as the most suitable method to establish animal model of ONT in our study, because it did not require sophisticated equipment, the toxic effects of ethanol could be controlled, results are highly reproducible and the surgery is relatively simple $[14,21]$, what's more important, it can imitate the pathological evolution of the early stage of natural osteonecrosis without adverse influence on the follow-up regeneration of bone tissue.

The macroscopic observation, X-ray, CT and histological examination have demonstrated that we have successfully induced the animal model of ONT from early to late stage, and revealed the pathological evolution of the osteonecrosis with time. The radiographic study is very important to the diagnosis of bone necrosis [7, 22]. In our study, different stage showed different radiographic presentation. At the early stage (no more than 12 weeks), the appearance of the cystic change in X-ray was very similar to those ONT of the early stage occurred in human being, however, it soon progressed to stage IV by the end of 24 weeks for the clear evidence of joint fusion. This phenomenon gave us a deep impression: without any intervention, the sheep's ONT of the early stage would generally deteriorate and the function of the ill ankle would be seriously impact for the reappearance of lameness 24 weeks postoperatively. The reason of the deterioration might lie in two aspects: the conspicuous osteopenia and the mass fibroplasias. The osteopenia was found out from the quantitative analysis of both the CT data and the histological study which indicating the cavity was gradually increasing and the area of living trabeculae dropped off with time. The fibroplasia was increasingly obvious in the histological examination as time went on. The combined effect of these two factors would lead to the decrease of talar mechanical properties and result in joint fusion at last.

Since the treatment on ONT of the late stage is very difficult, it is helpful to make certain the whole physiopathologic process of this animal model especially in the early stage. From the macroscopic observation and histological examination we could find that necrosis gradually occurred at the cancellous bone tissue, where some osteocytes began to die and the trabecula bones became loosen two weeks postoperatively, but the basic structure of the trabecula remains unchanged, therefore, this might be the initial phase of osteonecrosis and the major phenomenon was the destruction of intraosseous microcirculation. At week four postoperatively, large amount of osteocytes died and the trabecular structure was damaged considerably which resulted in the formation of cavity and the infiltration of segmental fibrous tissue, so bone destruction was the main feature in this period. Regeneration of bone tissue were observed at the 12th week, where the osteocytes repopulated and new bone tissue was deposited, moreover, empty lacunae decreased and proliferation of fibrous tissue sharply increased, which indicated this stage including both fibrous repair and bone reconstruction. This phase was very important for its two potential transformational directions, which meant bone reconstruction might dominate the following phase with proper intervention. On the contrary, without any treatment, infiltration of fibrous tissue would rule the pathological process which was closely connected with the joint fusion and indicated the poor prognosis. The bad ending was very obvious in almost every test methods in our study: the reappearance of moderate lameness, the increase of cavities both in the quantity and size, the formation of joint fusion, the infiltration of mass fibrous tissues. Therefore, it is necessary to take out certain treatment to prevent the deterioration in the early stage, and the suitable opportunity should no more than four weeks postoperatively in this animal model for the following reasons: (1) the living trabeculae began to decrease sharply; (2) the regeneration of the newborn bone became active; (3) the fibrous repair had started.

Although we have successfully established the clinically relevant animal model of ONT, there is one major limitation using this model for ONT study, which is that unlike human being as the bipedal animal, the biomechanics of talus in sheep, the quadrupedal animal, may be different from human being's. This is the general limitation for the current laboratory animals including rat, rabbit, minipigs, dog and sheep, which are all quadrupedal. Although, emu, a bipedal animal, could be used for developing the ONFH model [18], it is not suitable for ONT model, because emu does not have talus structure [23]. Despite of its quadrupedal feature, sheep possesses the following advantages as compared to other laboratory animal: (1) adult sheep have similar body weight to human being and the mechanical loading of its four limbs is well investigated, which is around half of the loading of human being, allowing relatively accurate prediction of the clinical scenario [24-26]; (2) the mineral component and the rate of metabolism and remodeling of the bone tissue in sheep are very close to 
human being [27-28]. As a result, sheep may become the most suitable animal models for ONT study currently.

In conclusion, we have successfully established a clinical relevant large animal model of ONT by intraosseous injection of pure ethanol. This preclinical animal disease model could imitate the pathological evolution of clinical osteonecrosis with the potential for the investigation on the underlying pathological mechanism and the exploration of diagnostics and treatment paradigms of aseptic osteonecrosis of human's talus.

\section{Acknowledgements}

This work was supported by the Natural Science Foundation of China (NSFC: 81171773). The authors acknowledge the technical help of Mr Rong Lv and Ms Jing Li at the Orthopaedic research laboratory of Xijing Hospital.

\section{Competing Interests}

The authors have declared that no competing interest exists.

\section{References}

1 Belal M, Ahmed S, Sandhu H, et al. Atraumatic avascular necrosis of the head of the talus. Foot and Ankle Surgery. 2004; 10: 213-5.

2 Delanois RE, Mont MA, Yoon TR, et al. Atraumatic osteonecrosis of the talus. J Bone Joint Surg Am. 1998; 80: 529-36.

3 Mont MA, Schon LC, Hungerford MW, et al. Avascular necrosis of the talus treated by core decompression. J Bone Joint Surg Br. 1996; 78(5): 827-30.

4 Reddy VRM, Selzer G, Faisal M, et al. Bilateral idiopathic avascular necrosis of talus: a case report. Foot and Ankle Surgery. 2003; 9: 35-9.

5 Guest M, Binfield PM, O'Doherty D. Core decompression of steroid induced avascular necrosis of the talus. The Foot. 1999; 9: 44-6.

6 Ficat RP. Idiopathic bone necrosis of the femoral head. J Bone Joint Surg Br. 1985; 67(1): 3-9.

7 Saini A, Saifuddin A. MRI of osteonecrosis. Clin Radiol. 2004; 59: 1079-93.

8 Assouline-Dayan Y, Chang C, Greenspan A, et al. Pathogenesis and natural history of osteonecrosis. Seminars in Arthritis and Rheumatism. 2002; 32(2): 94-124.

9 Levin D, Norman D, Zinman C, et al. Treatment of experimental avascular necrosis of the femoral head with hyperbaric oxygen in rats. Exp Mol Pathol. 1999; 67(2): 99-108.

10 Kilkenny C, Parsons N, Kadyszewski E, et al. Survey of the quality of experimental design, statistical analysis and reporting of research using animals. Survey of Animal Research. 2009; 4: e7824.

11 Wan L, de Asla RJ, Rubsh HE, et al. Determination of in-vivo articular cartilage contact areas of human talocrural joint under weightbearing conditions. Osteoarthritis Cartilage. 2006; 14(12): 1294-301.

12 Zhang $\mathrm{P}$, Liang $\mathrm{Y}$, Kim $\mathrm{H}$, et al. Evaluation of a pig femoral head osteonecrosis model. J Orthop Surg Res. 2010; 5: 15-21.

13 Li YL, Han R, Geng CK, et al. A new osteonecrosis animal model of the femoral head induced by microwave heating and repaired with tissue engineered bone. Int Orthop. 2009; 33(2): 573-80.

14 Manggold J, Sergi C, Becker K, et al. A new animal model of femoral head necrosis induced by intraosseous injection of ethanol. Lab Anim. 2002; 36(2): 173-80.

15 Bekler H, Uygur AM, Gökçe A, et al. The effect of steroid use on the pathogenesis of avascular necrosis of the femoral head: an animal model. Acta Orthop Traumatol Turc. 2007; 41(1): 58-63.

16 Vélez R, Soldado F, Hernández A, et al. A new preclinical femoral head osteonecrosis model in sheep. Arch Orthop Trauma Surg. 2011; 131(1): $5-9$.
17 Bejar J, Peled E, Boss JH. Vasculature deprivation--induced osteonecrosis of the rat femoral head as a model for therapeutic trials. Theor Biol Med Model. 2005; 2: 24.

18 Conzemius MG, Brown TD, Zhang Y, Robinson RA, et al. A new animal model of femoral head osteonecrosis: one that progresses to human-like mechanical failure. J Orthop Res. 2002; 20(2): 303-9.

19 Malizos KN, Quarles LD, Seaber AV, et al. An Experimental Canine Model of Osteonecrosis: Characterization of the Repair Process. J Orthop Res. 1993; 11(3): 350-7.

20 Wen $\mathrm{Q}, \mathrm{Ma} \mathrm{L}$, Chen YP, et al. A rabbit model of hormone-induced early avascular necrosis of the femoral head. Biomed Environ Sci. 2008; 21(5): 398-403.

$21 \mathrm{Zhu} \mathrm{ZH}$, Gao YS, Luo SH, et al. An animal model of femoral head osteonecrosis induced by a single injection of absolute alcohol: an experimental study. Med Sci Monit. 2011; 17(4): BR97-102.

22 Pearce DH, Mongiardi CN, Fornasier VL, et al. Avascular necrosis of the talus: a pictorial essay. Radiographics. 2005; 25(2): 399-410.

23 Main RP, Biewener AA. Skeletal strain patterns and growth in the emu hindlimb during ontogeny. J Exp Biol. 2007; 210(Pt 15):2676-90.

24 Newman E, Turner AS, Wark JD. The potential of sheep for the study of osteopenia: current status and comparison with other animal models. Bone. 1995; 16: 277-284.

25 Taylor WR, Heller MO, Bergmann G, et al. Tibio-femoral loading during human gait and stair climbing. J Orthop Res. 2004; 22: 625-32.

26 Taylor WR; Ehrig RM, Heller MO, et al. Tibio-femoral joint contact forces in sheep. J Biomech.2006; 39:791-8.

27 Reichert JC, Saifzadeh S, Wullschleger ME, et al. The challenge of establishing preclinical models for segmental bone defect research. Biomaterials. 2009; 30(12): 2149-63.

28 Ravaglioli A, Krajewski A, Celotti GC, et al. Mineral evolution of bone. Biomaterials. 1996; 17: 617-22. 